\title{
A novel method for analyzing the process of abrupt climate change
}

\author{
P. C. Yan ${ }^{1}$, G. L. Feng ${ }^{2,3}$, and W. Hou ${ }^{2}$ \\ ${ }^{1}$ College of Atmospheric Sciences, Lanzhou University, Lanzhou, China \\ ${ }^{2}$ National Climate Center, China Meteorological Administration, Beijing, China \\ ${ }^{3}$ College of Physical Science and Technology, Yangzhou University, Yangzhou, China
}

Correspondence to: W. Hou (houwei@cma.gov.cn)

Received: 17 December 2014 - Published in Nonlin. Processes Geophys. Discuss.: 20 January 2015

Revised: 6 April 2015 - Accepted: 7 April 2015 - Published: 4 May 2015

\begin{abstract}
A climate system which is transitioning from one state to another is known as an abrupt climate change. Most of the recent studies regarding abrupt climate change have focused on the changes occurring before and after the abrupt change point, while little attention has been given to the "transition process" which occurs when the system breaks away from the original state to a new state. In this study, a novel method for analyzing the process of abrupt climate change was presented. By using the mathematical model based on the logistic model, the process of the abrupt change could be analyzed and divided into different phases which include start moment, end moment, stable state, and unstable transition state. Meanwhile, the method was confirmed to be effective by testing in a study of Pacific decadal oscillation (PDO) time sequence, and the results of this study specify that this abrupt change process (ACP) of PDO has a relationship with global warming.
\end{abstract}

\section{Introduction}

The climate system is complex and chaotic (Shi, 2009). The abrupt climate change is described as the system transitioning from one stable state to another (Thom, 1972; Tong et al., 2014), i.e., the system swings between different states (Lorenz, 1976; Charney and DeVore, 1979), and it also has been verified in the climate system (Dai et al., 2012; Baker and Charlson, 1990; Wang et al., 2012; Alley et al., 2003; Xiao et al., 2011). Detection methods (Wan and Zhang, 2008; $\mathrm{Fu}$ and Wang, 1992; Yamamoto et al., 1986) have been greatly developed. Since the abrupt change theory was developed, increasing numbers of research (Goossens and Berger, 1986; Feng et al., 2008, 2011; Stefan, 2002) studies have been launched. Most of the current detection methods judge the abrupt change as the changing of the statistics, such as the mean, variance and trend in different moments. In order to clarify, such methods detect the abrupt change as 1 "point", and also the time sequence change occurring abruptly before and after the "abrupt change point" (He et al., 2012a, b).

Neither the external force nor the feedback mechanism can guarantee that the abrupt change occurs without a transition process, but the abrupt change must be obtained through a transition process (Li et al., 1996). Unfortunately, it seems that the transition process has not been mentioned in previous research. In this study, in order to determine when the onset, development, and extinction of the abrupt climate change events occur, a new novel detection method has been proposed. Through the definition of the transitional process, the abrupt climate change events were investigated as more than just a considered point, which may prove helpful in eliminating missed and false detection.

An "analytical method of abrupt change process" (AMACP), introduced by Yan et al. $(2013,2014)$, was proposed to objectively deal with the transition process. By means of giving the parameters actual physical meaning, the logistic model could be used to detect the transition process. This method is able to recognize the abrupt change process, which is different from the method detecting the "abrupt change point". In this study, the AM-ACP has been used to analyze the Pacific decadal oscillation (PDO) (http://jisao. washington.edu/pdo/) index. It was able to point out that the time sequence had several abrupt changes and the process had a relationship with global warming. Also, the PDO index started from 1900. 


\section{Theory on transition process of abrupt change}

The AM-ACP is based on the modified biologic modellogistic model (May, 1976) as shown in Eq. (1). This model showed the switching of the system among the different stable states, as shown in Fig. 1 (black line), and was able show the different degrees of abrupt change when the parameters of the model changed, as shown in Fig. 1 (gray dash line).

$\dot{x}=\kappa(x-\mu)(v-x), v<\mu$

In Eq. (1), the system remains in the stable state: $x=\mu$, or, $x=v$; when the rate of the system variable is zero, $\dot{x}=0$.

By using a numerical method, the relationship between the solution of the model, and the parameter $\kappa$ is shown in Fig. 2. In Fig. 2a, the system reached the same state $(x=\mu)$ during the evolution by changing the initial variable in threshold $\left(x_{0} \in(\nu, \mu)\right)$, when parameter $\kappa(\kappa=0.1)$ was positive (black lines). The dashed lines show the condition that the system reached in the state $x=v$, when the parameter $\kappa(\kappa<0)$ was negative. In Fig. $2 \mathrm{~b}$, the parameter $\kappa(\kappa=105)$ was larger than before, and the system became bifurcated. If the parameter $\kappa(\kappa=135)$ is much larger, the system will become chaotic as shown in Fig. 2c. Therefore, the parameter $\kappa$ is a stability parameter, and the parameters $\mu$ and $v$ are the start and end states before and after the abrupt change (Yan et al., 2012, 2013).

Figure 3 describes an abrupt change process when the system transforms from one stable state to another. In order to simplify the model, it was divided into three sections as shown in Fig. 3, and the length of each section is marked as $n_{1}, n_{2}$, and $n_{3}$, respectively.

1. In Sect. 1, during the period before the abrupt change, the system remained in the fixed stable state $x=v$.

2. In Sect. 2, the transition process, whereby the system transformed from a stable state $(x=v)$ to another state $(x=\mu)$, was considered to be linear. This means that the process could be fitted by the method of the least squares. The fitting equation is $x=h \cdot i+e$, where $h$ is the slope and reflects the rate of the amplitude over time during the transition process.

3. In Sect. 3, the system transformed to the stable state $(x=\mu)$ after the abrupt change, which is also represented as the equal of the sequence.

These parameters have been computed as a piecewise function:

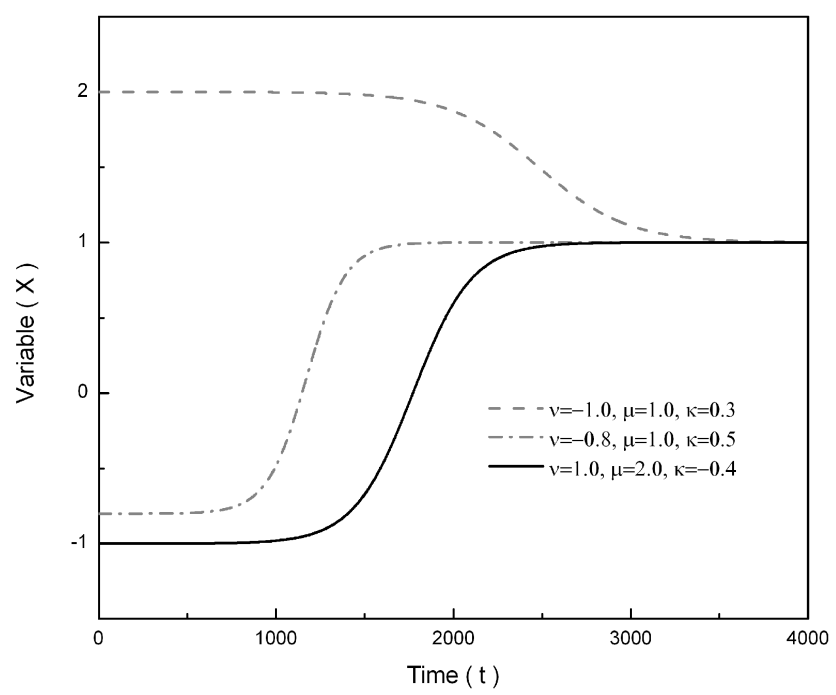

Figure 1. The evolution of the system over time, computed by rewriting Eq. (1) as its difference scheme: $x_{(t+1)}=\tau \kappa\left(x_{t}-\mu\right)\left(v-x_{t}\right)+x_{t}$, where $\tau=0.01$ is the time step.

$$
\left\{\begin{array}{l}
v=\sum_{i=1}^{n_{1}} x_{i} / n_{1} \\
h=\sum_{i=n_{1}+1}^{n_{1}+n_{2}} \bar{i} \cdot \bar{x}_{i} / \sum_{i=n_{1}+1}^{n_{1}+n_{2}} \bar{i}^{2}, e=h \cdot \bar{x}_{i}-\bar{i} \\
\mu=\sum_{i=n_{1}+n_{2}+1}^{n_{1}+n_{2}+n_{3}} x_{i} / n_{3}
\end{array}\right.
$$

In Sect. 2, the linear transition process has been marked as a black line. It is fixed by two points, $\mathrm{A}$ and $\mathrm{B}$, expressed as $\left(x_{\alpha}, t_{\alpha}\right)$ and $\left(x_{\beta}, t_{\beta}\right)$, respectively. Then, the slope of the linear equation is expressed as

$h=\frac{x_{\alpha}-x_{\beta}}{t_{\alpha}-t_{\beta}}$.

The location parameters $\alpha$ and $\beta$ have been defined to describe the location of these two points as follows:

$\left\{\begin{array}{l}x_{\alpha}=\alpha(\mu-v)+v \\ x_{\beta}=\beta(\mu-v)+v\end{array}\right.$.

The parameter $\alpha$ (or $\beta$ ) is the ratio of the distance that the point deviates from the start state and the amplitude of the abrupt change; then, $\alpha \in[0,1], \beta \in[0,1]$. 
a

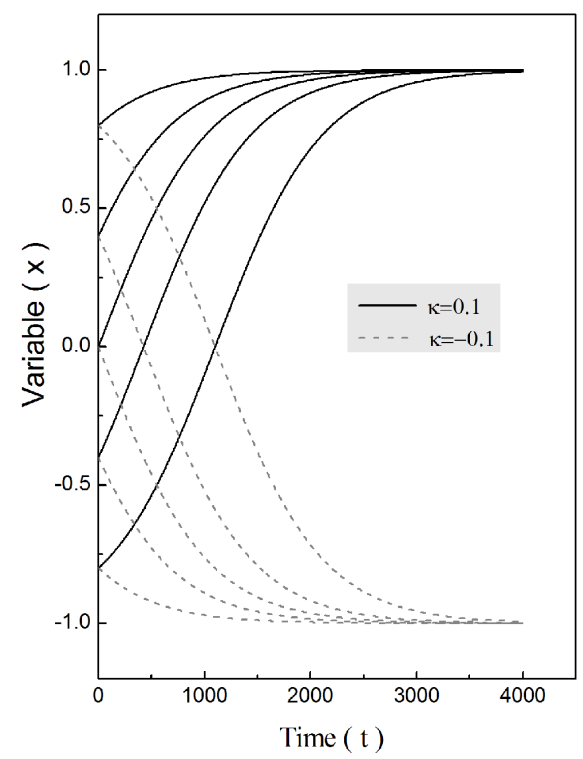

b
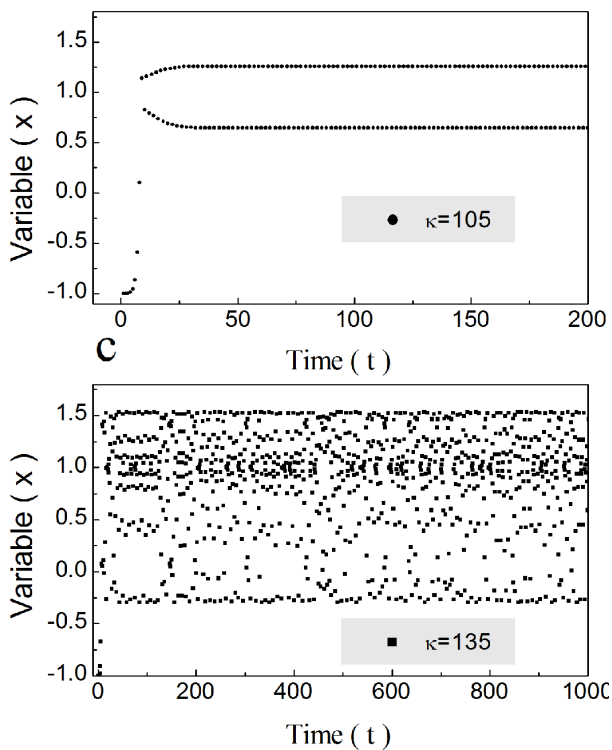

Figure 2. The evolution of the system over time, with different stability parameters: (a) the system reaches to the stable states with a different initial variable when parameter $\kappa= \pm 0.01$; (b) the system becomes bifurcated when the parameter $\kappa=105$; (c) the system becomes chaotic when the parameter $\kappa=135$.

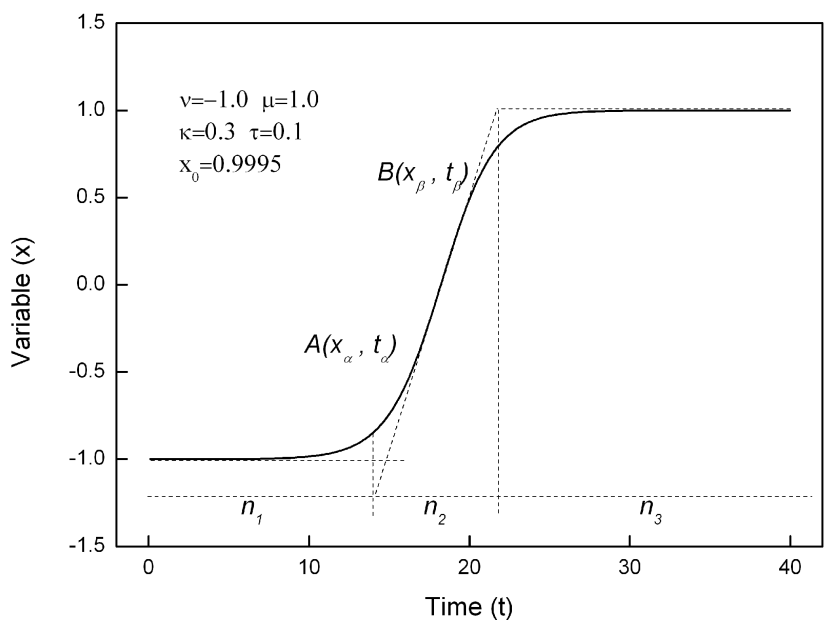

Figure 3. The abrupt change process and its sections. The three sections are as follows: period before the abrupt change, period of abrupt change process, and period after abrupt change.

Integral Eq. (1),

$$
\begin{gathered}
\frac{1}{\mu-v} \int_{x_{0}}^{x}\left(\frac{1}{x-v}-\frac{1}{x-\mu}\right) \mathrm{d} x=\kappa \int_{t_{0}}^{t} \mathrm{~d} t \\
\Rightarrow \ln \left(\frac{x-v}{x-\mu} \cdot \frac{x_{0}-\mu}{x_{0}-v}\right)=\kappa(\mu-v)\left(t-t_{0}\right) \\
\Rightarrow \frac{x-v}{x-\mu}=\frac{x_{0}-v}{x_{0}-\mu} \cdot e^{\kappa(\mu-v)\left(t-t_{0}\right)} .
\end{gathered}
$$

Using the intermediate variable $\xi=\frac{x_{0}-v}{x_{0}-\mu} \cdot e^{\kappa(\mu-v)\left(t-t_{0}\right)}=$ $\frac{x-v}{x-\mu}$; then,

$t=t_{0}+\frac{1}{\kappa(\mu-v)} \ln \left(\frac{x_{0}-\mu}{x_{0}-v} \cdot \xi\right)$.

Equation (3) is expressed by

$$
\begin{aligned}
h & =\frac{\alpha(\mu-v)-\beta(\mu-v)}{\frac{1}{\kappa(\mu-v)}\left(\ln \left(\frac{x_{0}-\mu}{x_{0}-v} \cdot \xi_{\alpha}\right)-\ln \left(\frac{x_{0}-\mu}{x_{0}-v} \cdot \xi_{\beta}\right)\right)} \\
& =\kappa(\mu-v)^{2} \frac{\alpha-\beta}{\ln \left(\xi_{\alpha} / \xi_{\beta}\right)} \\
& =\kappa(\mu-v)^{2} \frac{\alpha-\beta}{\ln \left(\frac{\alpha}{\beta} \cdot \frac{1-\beta}{1-\alpha}\right)} .
\end{aligned}
$$

A new parameter is defined as $\chi=\frac{\alpha-\beta}{\ln \left(\frac{\alpha}{\beta} \cdot \frac{1-\beta}{1-\alpha}\right)}$, which has a relationship with the location parameters $\alpha$ and $\beta$. The relationship is shown in Fig. 4, and parameter $\chi$ is almost constant when the values of $\alpha$ and $\beta$ are within a certain range. In this paper, $\alpha=0.2, \beta=0.8$, and $\chi=0.2164$.

Equation (7) is rewritten as follows:

$h=\kappa(\mu-v)^{2} \chi$.

As it is known that $\mu-v$ is the amplitude during the abrupt change, Eq. (8) illustrates the quantitative relationship among the rates, the amplitude, and the stability parameter of the abrupt change process. 
Therefore, by changing the lengths $n_{1}, n_{2}$, and $n_{3}$, the piecewise Eq. (2) could then be used to optimally fit an abrupt change process with a group of parameters $\varphi(h, \mu, \nu$, $n_{1}, n_{2}, n_{3}$ ) from a sequence which occurred during a similar abrupt change.

By testing a sub-sequence obtained from an entire time sequence, a group of parameters $\varphi\left(h, \mu, v, n_{1}, n_{2}, n_{3}\right)^{1}$ could then be calculated. Also, by moving the sub-sequence onto the entire time sequence (as shown in Fig. 5a), a series of groups of parameters $\left(\varphi^{1}, \varphi^{2}, \varphi^{3}, \cdots, \varphi^{m}\right)$ could then be calculated. The process of the abrupt change could then be studied by analyzing the parameters. In Fig. 5b, in order to count the start moment $v$, the two states $(v=2.0$ and $v=4.0)$ are detected with higher frequency. It should be noticed that the reason the frequency of state 2 is higher than state 4 is only because the start moment (just like state 2 ) is counted here. In contrast, the frequency of state 4 will be higher than state 2 , if only the end moment (just like state 4 ) is counted. To clarify, this means that two stable states exist in the time sequence, i.e., the system presents a double-peak structure. With regards to the relationship between the detecting moment and the start moment, as shown in Fig. 5c (blue line), the same start moment has always been detected over a long period, with the detecting moment changing from point 100 to point 500. That start moment is calculated (red line) in Fig. 5c. At point 150, the frequency is much higher than in the others. The reason being that the point is the start moment of the abrupt change process of the ideal model. By utilizing the frequency of the start moment to judge the abrupt change, this method would improve the situations of missing and false detection, which were happening when using the traditional method. Figure $5 \mathrm{~d}$ is a phase diagram of the "start-end" states, and each point in the figure represents a detection result: the $x$ axis is the start state, and the $y$ axis is the end state.

In the vertical line (the red line), the points present a process that the start state stays constant, and the end state increases. This indicates that the sub-sequence has passed by the start moment during the moving process. Therefore, the vertical line represents a process in which the system deviates from a stable state.

In the horizontal line (the blue line), the points represent a process that the start state is increasing, and the end start state remains constant. This indicates that the sub-sequence has passed by the end moment during the moving process, which indicates that the horizontal line represents a process that the system is approaching a stable state.

The green line, which runs parallel to the diagonal, shows that the sub-sequence is in the process of an abrupt change, since both the start state and the end state are increasing, and the difference between them is constant.

It should be noted that the line running parallel to the diagonal would vanish if the length of the sub-sequence is larger than the length of the abrupt change process. Also, the vertical line and the horizontal would still exist.

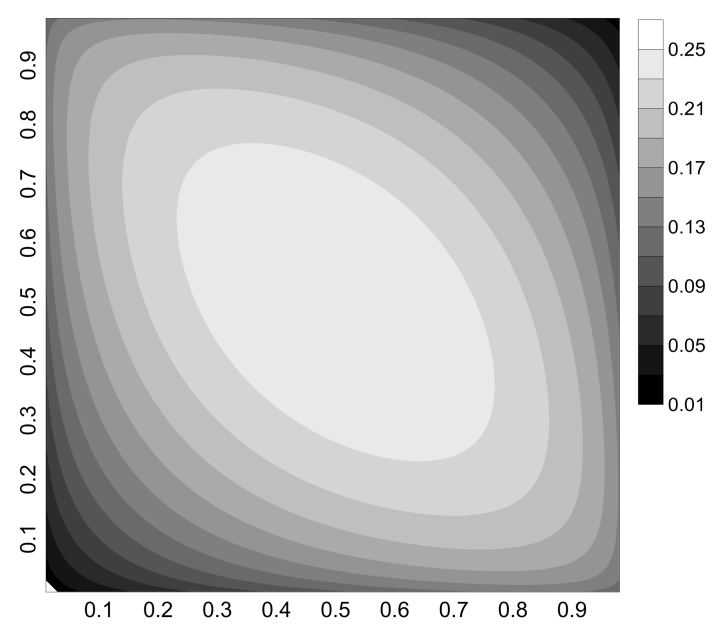

Figure 4. The relationship parameter $\chi$ and parameters $\alpha, \beta$, where the $x$ axis is parameter $\alpha$, the $y$ axis is parameter $\beta$ and the contour is parameter $\chi$.

Therefore, based on the analysis of the result of the detection, the AM-ACP can be used to effectively detect the process of the abrupt change. In future studies, this method will be used to detect a real-time sequence, and the statistical characters would then be shown in order to study the abrupt change process.

\section{Characteristics of abrupt change process of Pacific decadal oscillation}

In this study, based on the theory of the AM-ACP, the PDO index has been analyzed to study the abrupt change process. The PDO has been considered as an important indicator of the decadal variability of the Pacific Ocean (Meehl et al., 1993), which has a strong relationship with the climate of China (Wang et al., 2013). It has been shown in many previous studies, that this index has experienced several abrupt changes over the past 100 years (Mantua et al., 1997; Tsonis et al., 2007), and that all of these abrupt changes correspond to the global climate changes.

During the application of AM-ACP on the PDO index, parameters $\mu$ and $v$ represent the states before and after the abrupt change process, respectively. Parameter $\kappa$ still represents the stability of the PDO index; a larger $\kappa$ means more stability and vice versa (Yan et al., 2012, 2013). Therefore, many families of parameters could be obtained by moving the sub-sequence whose length is set at 10 years, such as what has been done to the ideal model in Sect. 2. By analyzing many families of parameters, the statistical distribution of the start state was determined and is illustrated in Fig. 6. During the calculation, the length of the sub-sequence was set at 10 years (Fig. 6a), 20 years (Fig. 6b), 30 years (Fig. 6c), and 40 years (Fig. 6d). All four of these frequency distributions had two peaks, which means the system was mainly dis- 

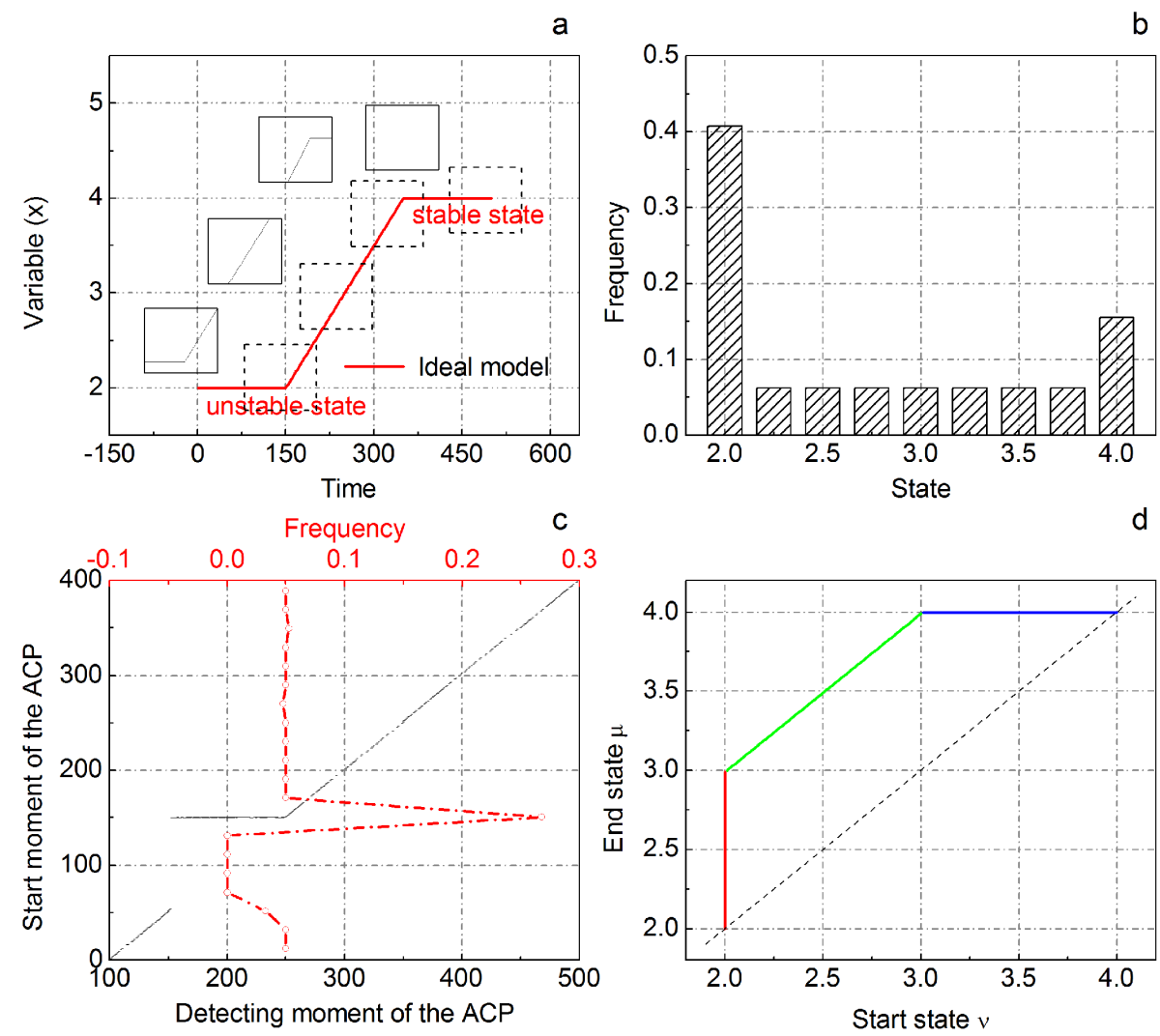

Figure 5. The detection of the abrupt change process of the ideal model and the analysis of the results. (a) The ideal model (red line) and its detection of the abrupt change process in different observed windows (black lines) with sub-sequences; (b) the statistics of the start states; (c) the relationship between the detecting moment and the start moment (blue line), and the frequency of the star moment (red line); (d) the phase diagram of "start-end" states.

tributed on two stable states, as illustrated in Fig. 5b. Furthermore, under certain conditions, the system may have crossed over from one state to another. Such a structure has been verified several times in the climate system (Goldblatt et al., 2006; Alexander et al., 2012; Zerkle et al., 2012).

In Fig. 7, it is shown that the different start moments of the abrupt changes in the PDO index time sequence have been detected at different detecting moments. The results showed that the frequency of the abrupt change start in some years was much higher than in other years. Also, when the length of sub-sequence was set as 40 years, as shown in Fig. 7d, then the start moments of the abrupt changes that started in 1940 and 1976 were detectable for a rather long time period (detecting moment). These results are precisely when two transitions occurred. These transitions were when the PDO index time sequence transferred from a positive phase to a negative phase in 1940, and from a negative phase to a positive phase in 1976 (Francis and Hare, 2007). The abrupt change in 1955, was detected by an abnormally smaller value of the PDO index time sequence in that year. In addition, the frequency of detected abrupt changes in 1934 and 1988 was higher, which indicated that the system did deviate, but with only a small amplitude, from a stable state.

Figure 8 shows the "start-end states" phase diagram of the PDO system during the period from 1900 to 2010, and the five clear abrupt changes are marked with five different colors in the diagram. As shown in Fig. 5d, the vertical and horizontal lines represent different sections of the abrupt change process. In regards to the different lengths of the sub-sequence, all five abrupt change processes have been detected. By taking a 30-year length as the set time length for example, the result was analyzed as follows.

The first abrupt change process started in 1934 (marked with blue dots), and the vertical section line which consists of blue dots in the phase diagram, is located on the left-hand side of the diagonal line. By comparing this with Fig. 5d, we concluded that this was a continuously increasing process, and had increased to a new stable state.

The second abrupt change process started in 1940 (marked with light blue dots), and it is the vertical line located on the right-hand side of the diagonal line. By comparing this with Fig. 5d, we concluded that this indicated a decreasing process from a stable state. 

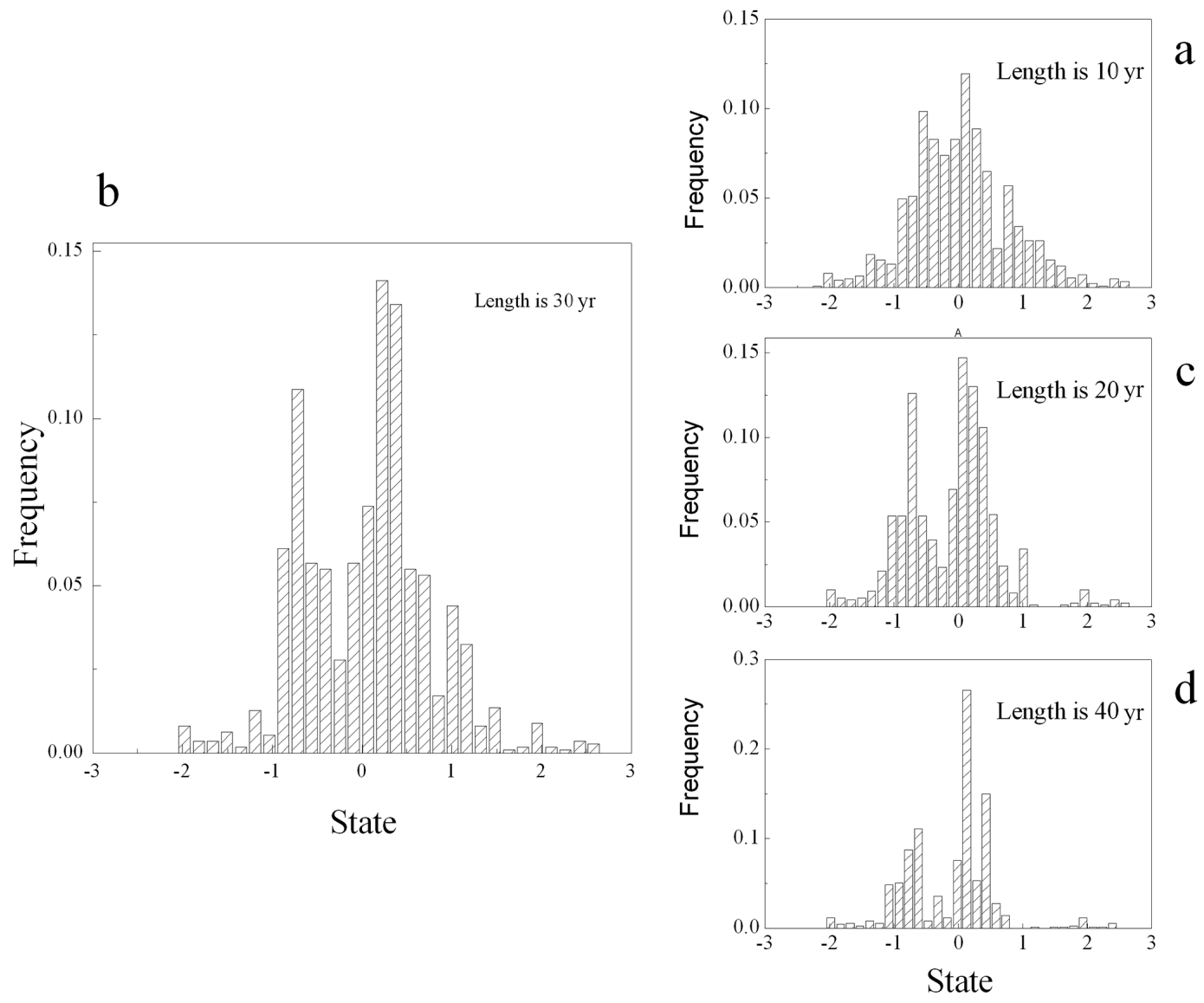

Figure 6. Histogram of statistical distribution of the start state. The $x$ axis refers to the statistical interval for the PDO index, and the $y$ axis refers to the frequency.

The third abrupt change process started in 1955 (marked with green dots), and it is located in a neighboring area of the diagonal line. By comparing this with Fig. 5d, we concluded that this was a non-abrupt change process, for example, abnormally small values had been detected in the original time sequence.

The fourth abrupt change process started in 1976 (marked with yellow dots), and the red dots consist of a vertical line located on the left-hand side of the diagonal line. This indicates an increasing process to a stable state, which is similar to the abrupt change that started in 1934 marked with a blue line.

The fifth abrupt change process started in 1988 (marked with red dots), which has been divided into two sections: an approximate vertical and horizontal lines, where the vertical line indicates a decreasing process from an stable state, and the horizontal line indicates a decreasing process to an stable state.

In conclusion, the dotted line in the "start-end states" diagram indicates different abrupt change processes which may be included in certain stages of an abrupt change. For all of the abrupt changes detected, two changes which started in
1940 and 1976 have longer vertical lines, indicating these two abrupt changes were more severe. This is the reason why these two abrupt changes have also been detected by some other detection methods.

In Fig. 9, some further research is shown concerning the relationship between the persist time and the start moment of the abrupt change processes. The results indicated that the abrupt changes starting from different moments have clear differences in persist times. The abrupt changes which started in 1940 and 1976, continued for 136 and 144 months, respectively. This is consistent with the observations of the time sequence of the PDO index. The abrupt change which started in 1988, continued for 120 months; the abrupt change started in 1934 , continued for 70 months; and the abrupt change started in 1955, only continued for 38 months. In addition, by sliding the sub-sequence onto the entire time sequence, the persist time of each abrupt change may be detected more than once, i.e., the abrupt changes started in 1940 and 1976. The reason for this multiple detection is that such abrupt changes belong to different abrupt change processes.

To further inspect the relationship between the start moment of the abrupt changes and their persist time as shown in 


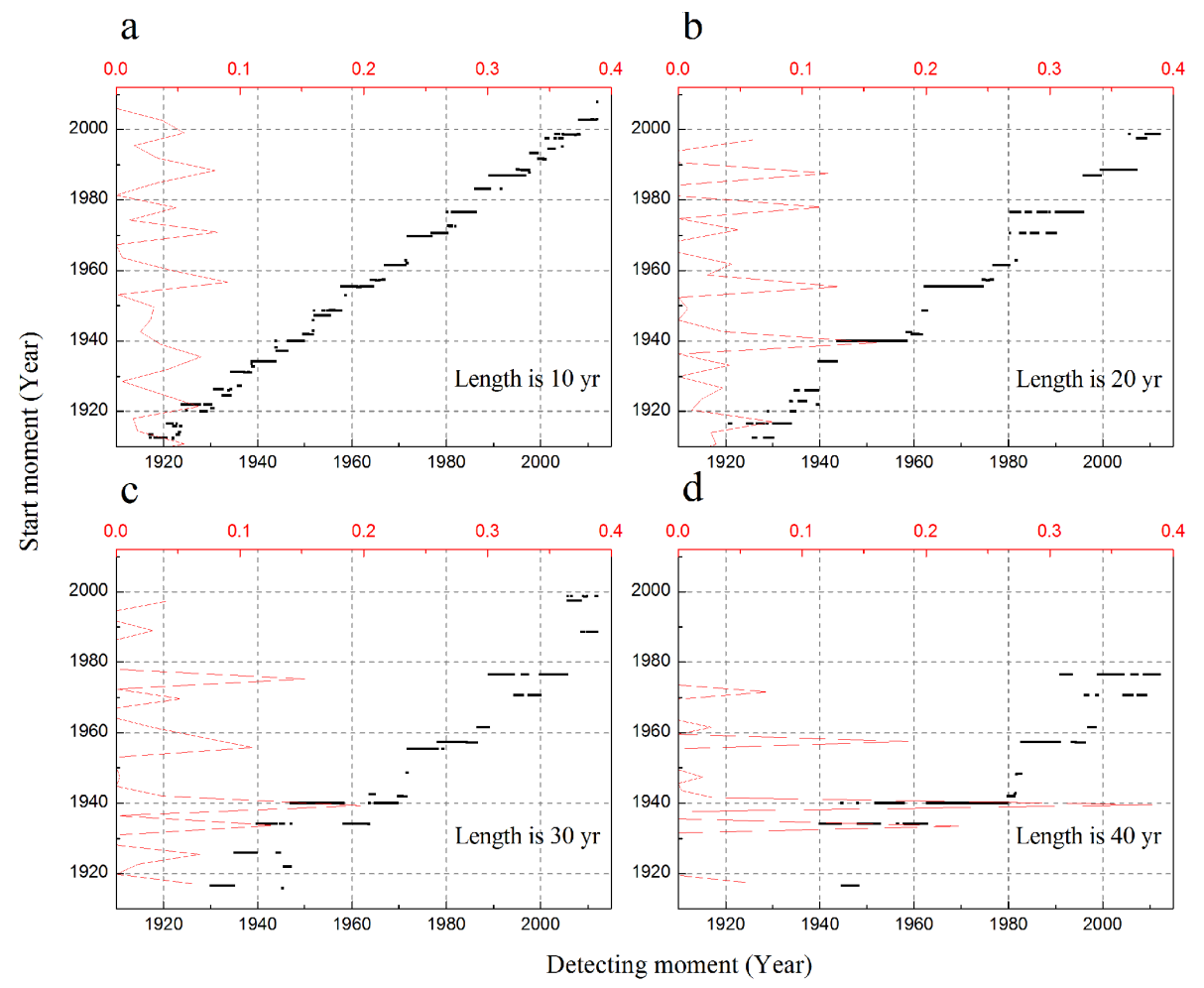

Figure 7. Start moment of the abrupt change in PDO index series with different detecting moments. The $x$ axis refers to the detecting moment of each sub-sequence detected, or the moment for detecting the time sequence, while the $y$ axis refers to the start moment. The black short line indicates the start moment of the abrupt change, which is detectable in a rather long time period; and the red line refers to the frequency of the start moment. (a-d) are the different conditions in which the lengths of sub-sequence are set as 10a, 20a, 30a, and 40a.

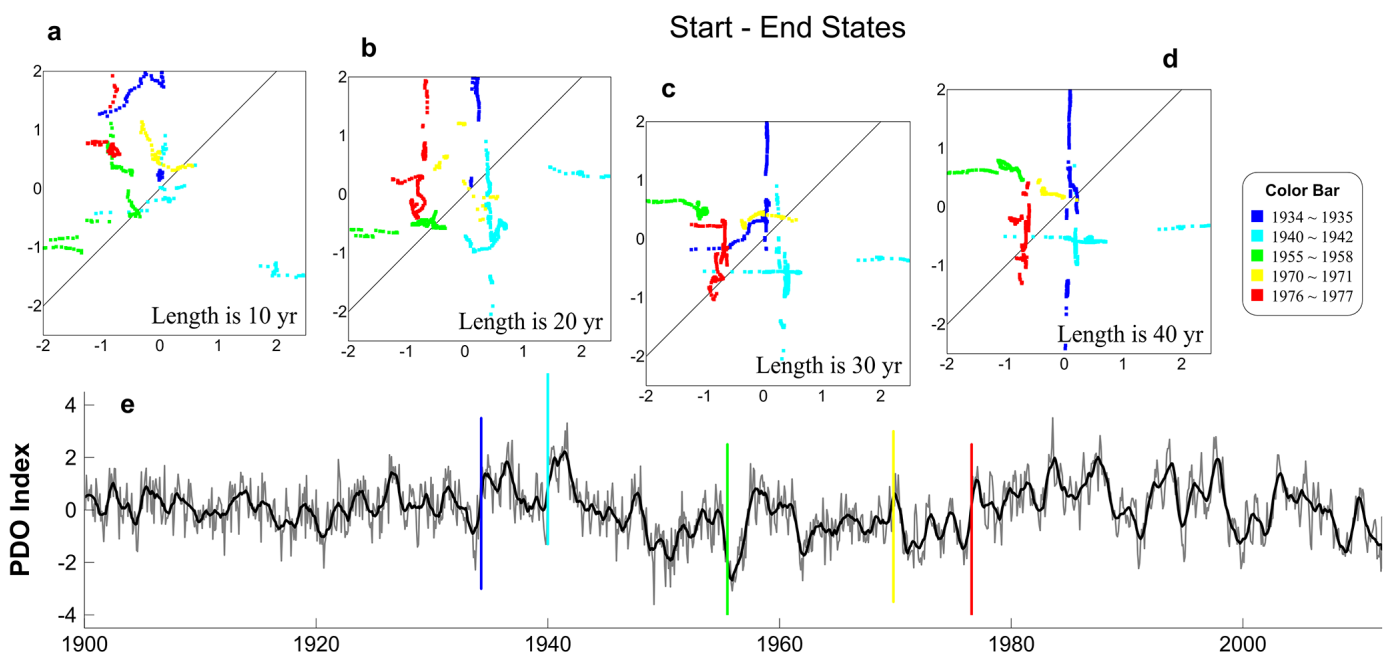

Figure 8. The PDO index and its "start-end states" phase diagram. (a-d) are the phase diagrams: the $x$ axis refers to start state of the abrupt change detected through each sub-sequence, the $y$ axis refers to the end state, and each point in the figure refers to one detection; the different colors refer to different processes with different start moments of abrupt change. (e) is the PDO index: the $x$ axis refers to time and the $y$ axis refers to the index. 


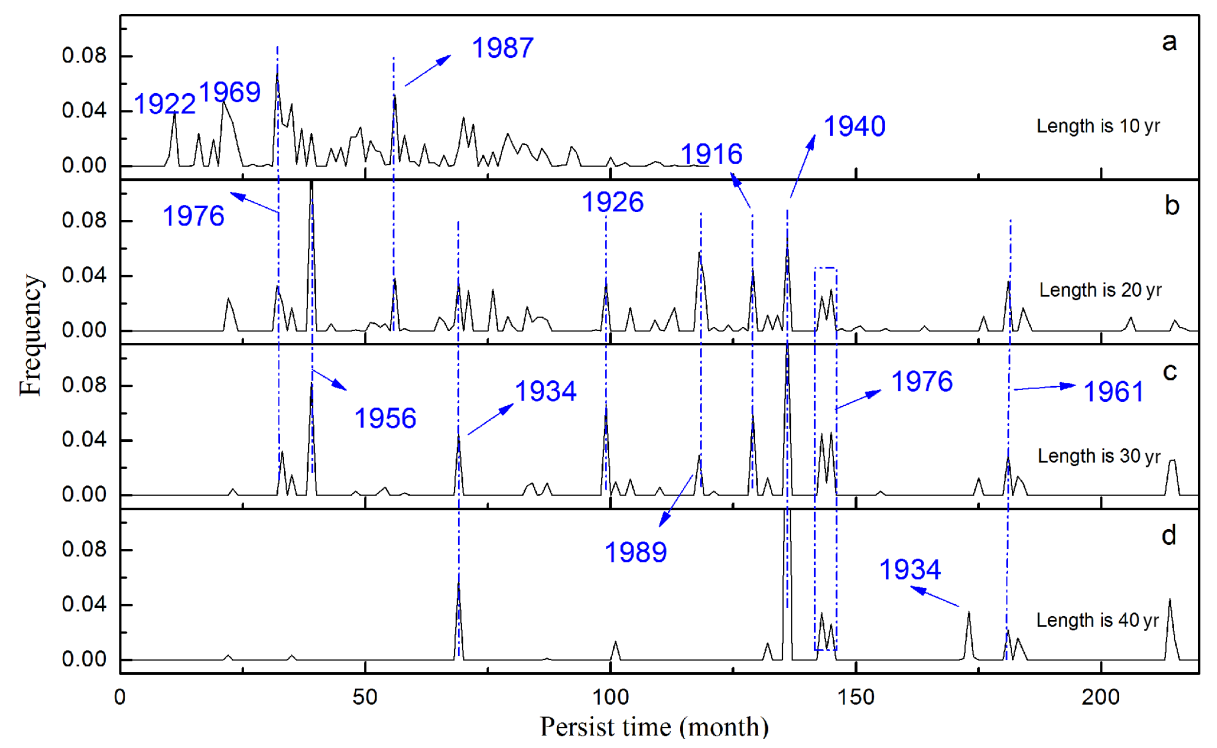

Figure 9. Distribution of the abrupt change persist time with the start moment. (a-d) show the different conditions in which the length of the sub-sequence are set, which are 10a, 20a, 30a, and 40a. The $x$ axis refers to the persist time of the abrupt change, the $y$ axis refers to the frequency of the abrupt changes that satisfies the conditions, and the characters mark the start moments of each abrupt change process.

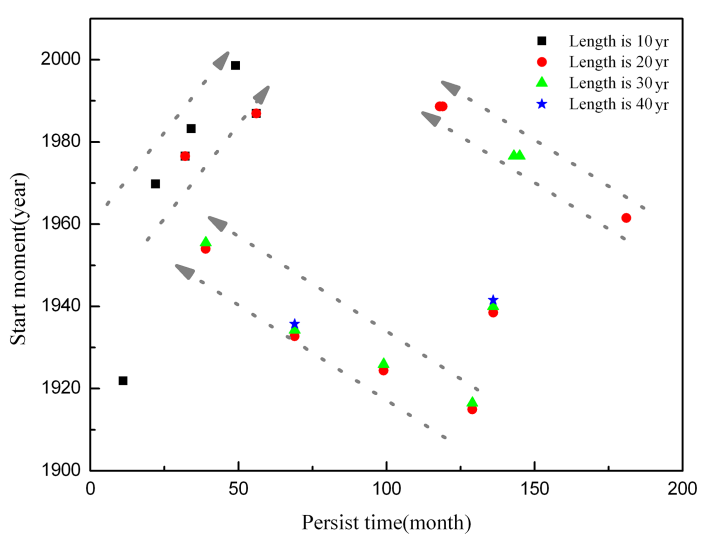

Figure 10. Abrupt changes start at different moments than their persist times. The $x$ axis refers to the persist time of each abrupt change process, and the $y$ axis refers to the start moment.

Fig. 10, the distribution of the relationship is shown with the length of sub-sequence set as 10,20,30, and 40 years. The number of abrupt changes following the 1960s, significantly exceeded that before the 1960s, which reflects the fact that the climate system might have become progressively unstable. In regards to the several abrupt changes occurring before the 1960s, the later the abrupt change started, the shorter the persist time of the change became. As for the abrupt changes after the 1960s, these abrupt changes can be divided into two groups, which are those lasting for more than 100 months, and those lasting for less than 50 months. In regards to the persist times, in the former group it was found to be shorter, while in the latter group the persist time was extended, and the two groups were in a symmetrical distribution for approximately 70 to 80 months. In addition, the global temperature continued to increase prior to 1940 , and reached its peak value during the 1940s, then it remained at a lower level for the following 10 years, before beginning to slowly rise at the start of the 1950s (IPCC, 2007). This is consistent with the abrupt change in the PDO index time sequence. These facts indicated that, under the context of global warming, the oceanic system showed an evidently unstable state, and that the persist time of the abrupt changes which began after the 1950 s presented a trend of becoming closer to the range of 70 to 80 months.

\section{Conclusions}

As the evidence of a climate system swing among different states has undoubtedly given us an opportunity to learn more about abrupt climate change events, a new method to analysis the process of abrupt climate change, AM-ACP, was proposed in this study, and the method has also provided a new perspective to research abrupt climate change.

By using an ideal mean change time sequence to simulate the process of the abrupt change, there are three characteristics that can be shown: (1) multi-stable states exist in the abrupt change events, (2) the start moment of the abrupt change is detectable in different times, and (3) the "start-end states" phase diagram could be used to express the duration of the abrupt change.

Moreover, these characteristics also could be obtained as the AM-ACP is applied to the PDO index. The results shown that the ocean system is bi-stability, and the transi- 
tion among different stable states has occurred five times over the past 100 years in 1934, 1940, 1955, 1970, and 1976, and the "start-end states" phase diagrams show the transformation processes clearly. In addition, the persist time of each abrupt change is almost constant, and the persist time becomes shorter with global warming. After 1960s, the persist time gets longer when shorter than 100 months; on the contrary, the persist time gets shorter when it is longer than 100 months. With regards to the process of abrupt climate change, further work is needed to research this subject, and more evidence concerning abrupt climate change is needed in order to find a more efficacious method to research the abrupt change process. Now we may unable to accurately forecast exactly when an abrupt climate change will occur, but at least we hope that in the future we can devise a methodology which can be used to determine whether we are in the process of an abrupt climate change, which is of great importance to human society.

Acknowledgements. The authors would like to thank the anonymous reviewers and the editors for helpful suggestions. This project was supported by National Natural Science Foundation of China (grant nos. 41175067 and 41305056), National Basic Research Program of China (grant no. 2012CB955901), and National Natural Science Foundation of China (grant no. 41375069).

Edited by: V. Perez-Munuzuri

Reviewed by: two anonymous referees

\section{References}

Alexander, R., Reinhard, C., and Andrey, G.: Multistability and critical thresholds of the Greenland ice sheet, Nat. Clim. Change, 2, 429-432, 2012.

Alley, R. B., Marotzke, J., Nordhaus, W. D., Overpeck, J. T., Peteet, D. M., Pielke, R. A., Pierrehumbert Jr., R. T., Rhines, P. B., Stocker, T. F., Talley, L. D., and Wallace, J. M.: Abrupt climate change, Science, 299, 2005-2010, 2003.

Baker, M. B. and Charlson, R, J.: Bistability of CCN concentrations and thermodynamics in the cloud-topped boundary layer, Nature, 345, 142-145, 1990.

Charney, J. G. and DeVore, J. G.: Multiple flow equilibria in the atmosphere and blocking, J. Atmos. Sci., 36, 1205-1216, 1979.

Dai, X. G., Wang, P., and Zhang, K. J.: A decomposition study of moisture transport divergence for inter-decadal change in East Asia summer rainfall during 1958-2001, Chin. Phys. B, 21, 119201, doi:10.1088/1674-1056/21/11/119201, 2012.

Feng, G. L., Gong, Z. Q., and Zhi, R.: Latest advances of climate change detecting technologies, Acta Meteorol. Sin., 66, 892905, 2008.

Feng, G. L., Hou, W., Zhi, R., Yang, P., Zhang, D. Q., Gong, Z. Q., and Wan, S. Q.: Research on Detecting, Diagnosing and Predictability of Extreme Climate Events, Science Press, Beijing, China, 2011.
Francis, R. C. and Hare, S. R.: Decadal-scale regime shifts in the large marine ecosystems of the Northeast Pacific: a case for historical science, Fish. Oceanogr., 3, 279-291, 2007.

$\mathrm{Fu}, \mathrm{C}$. B. and Wang, Q.: The definition and detection of the abrupt climate change, Scient. Atmos. Sin., 16, 482-493, 1992.

Goldblatt, C., Lenton, T. M., and Watson, A. J.: Bistability of atmospheric oxygen and the Great Oxidation, Nature, 443, 683-686, 2006.

Goossens, C. and Berger, A.: Annual and seasonal climatic variations of northern hemisphere and europe over the last century, Ann. Geo-Phys., 4, 385-400, 1986.

He, W. P., Feng, G. L., Wu, Q., He, T., Wan, S. Q., and Chou, J. F.: A new method for abrupt dynamic change detection of correlated time series, Int. J. Climatol., 32, 1604-1614, doi:10.1002/joc.2367, 2012a.

He, W. P., Wan, S. Q., Jiang, Y. D., Zhang, W., Wu, Q., and $\mathrm{He}$, T.: Detecting abrupt change on the basis of skewness: Numerical tests and applications, Int. J. Climatol., 33, 2713-2727, doi:10.1002/joc.3624, $2012 b$.

IPCC: Climate Change 2007, Synthesis Report, Cambridge University Press, Cambridge, UK, 2007.

Li, J. P., Chou, J. F., and Shi, J. E.: Complete definition and types of abrupt climate change, J. Beijing Meteorol. Coll., 1, 7-12, 1996.

Lorenz, E. N.: Nondeterministic theories of Climate change, Quaternary Res., 6, 495-506, 1976.

Mantua, N. J., Hare, S. R., Zhang, Y., Wallace, J. M., and Francis, R. C.: A Pacific interdecadal climate oscillation with impacts on salmon production, B. Am. Meteorol. Soc., 78, 1069-1079, 1997.

May, R.: Simple mathematical models with very complicated dynamics, Nature, 261, 459-467, 1976.

Meehl, G. A., Branstator, G. W., and Washington, W. M.: Tropical Pacific Interannual Variability and $\mathrm{CO}_{2}$ Climate Change, J. Climate, 6, 42-63, 1993.

Shi, N.: Meteorological statistical forecast, Meteorological Press, Beijing, China, 2009.

Stefan, R.: Ocean circulation and climate during the past 120,000 years, Nature, 419, 207-214, 2002.

Thom, R.: Stability Structural and Morphogenesis, Sichuan Education Press, Sichuan, China, 1972.

Tong, J. L., Wu, H., Hou, W., He, W. P., and Zhou, J.: Early warning signals of abrupt temperature change in different regions of China over the past 50 years, Chin. Phys. B, 23, 049201, doi:10.1088/1674-1056/23/4/049201, 2014.

Tsonis, A. A., Swanson, K., and Kravtsov, S.: A new dynamical mechanism for major climate shifts, Geophys. Res. Lett., 34, L13705, doi:10.1029/2007GL030288, 2007.

Wan, S. Q. and Zhang, W.: Detection and attribution of abrupt climate changes in the last one hundred years, Chin. Phys. B, 17, 2311-2316, 2008.

Wang, K., Feng, G. L., Zeng, Y. X., and Wang, X. J.: Analysis of Stable Components in Extended-Range Forecast for the Coming 10-30 Days in Winter 2010 and 2011, Chin. Phys. B, 22, 129202, doi:10.1088/1674-1056/22/12/129202, 2013.

Wang, X. J., Zhi, R., He, W. P., and Gong, Z. Q.: Collective behaviour of climate indices in the North Pacific air-sea system and its potential relationships with decadal climate changes, Chin. Phys. B, 21, 029201, doi:10.1088/1674-1056/21/2/029201, 2012. 
Xiao, D., Li, J. P., and Zhao, P.:Four-dimensional structures and physical process of the decadal abrupt changes of the northern extratropical ocean-atmosphere system in the 1980s, Int. J. Climatol., 32, 983-994, doi:10.1002/joc.2326, 2011.

Yamamoto, R., Iwashima, T., and Sanga, N. K.: Climatic jump,a hypothesis in climate diagnosis, Meteorol. Soc. Jpn., 63, 11571160, 1986.

Yan, G. H., Yan, P. C., Hou, W., and Wu, H.: A method of abrupt change process analysis based on Logistic model and its applications, Acta Phys. Sin., 62, 079202, doi:10.7498/aps.62.079202, 2013.
Yan, P. C., Hou, W., and Hu, J. G.: The critical warning research of the mean time sequence mutations based on Logistic model, Acta Phys. Sin., 61, 189202, doi:10.7498/aps.61.189202, 2012.

Yan, P. C., Feng, G. L., Hou, W., and Wu, H.: Statistical characteristics on decadal abrupt change process of time sequence in $500 \mathrm{hPa}$, Chin. J. Atmos. Sci., 38, 861-873, 2014.

Zerkle, A. L., Claire, M. W., Domagal-Goldman, S. D., Farquhar, J., and Poulton, S. W.: A bistable organic-rich atmosphere on the Neoarchaean Earth, Nat. Geosci., 5, 359-363, 2012. 